\title{
Closed-form approximations and series representations for partially saturated ocean acoustic processes
}

\author{
Anastassios N. Perakis \\ Department of Naval Architecture and Marine Engineering, The University of Michigan, Ann Arbor, \\ Michigan 48109 \\ Efstratios Nikolaidis \\ Department of Aerospace and Ocean Engineering, Virginia Polytechnic Institute and State University, \\ Blacksburg, Virginia 24061
}

\begin{abstract}
Emmanuel Katzouros ${ }^{\text {a) }}$
Department of Naval Architecture and Marine Engineering, The University of Michigan, Ann Arbor, Michigan 48109
\end{abstract}

(Received 30 July 1986; accepted for publication 24 November 1987)

\begin{abstract}
An approximate, closed-form expression for the value of the integral encountered in the calculation of the probability density function (PDF) of the envelope of a partially saturated ocean acoustic process is obtained. Furthermore, an expression of this PDF as a series of modified Bessel functions is presented. The results may also be directly applied to the evaluation of the PDF encountered in the structural reliability analysis of rotating machinery components. Numerical applications show that the closed-form expression is always within $1 \%-2 \%$ of the exact result. The required computational effort is substantially lower than that required by direct numerical integration.
\end{abstract}

PACS numbers: 43.60.Cg, 43.30. $-\mathrm{k}$

\section{INTRODUCTION}

Acoustic signals in the ocean environment that propagate through multiple independent paths form processes named unsaturated, fully saturated, and partially saturated. ${ }^{1,2}$ When the source-receiver distance is small, and for frequencies of the process such that negligible perturbations occur between paths, the acoustic propagation is called unsaturated. In this case, the phases of the paths are constant. On the other extreme, when the source-receiver distance is large enough, and/or for high frequencies, the phases of the propagation paths can be characterized as independent, uniformly distributed random variables with standard deviations equal to $2 \pi$ or Gaussian distributed with standard deviation $\geqslant 2 \pi$. In that case, the process is called fully saturated. The term partially saturated refers to a narrow-band multipath acoustic process occurring at intermediate ranges of the source-receiver distance. In that case, the phases of the channels can be characterized as independent random variables with standard deviations less than $2 \pi$. For a more detailed discussion of the above definitions, see Ref. 1.

Knowledge of certain statistical properties of the acoustic signal envelope is required in order to use existing signal detection algorithms. ${ }^{3}$ The first- and second-order PDFs of the envelope of a partially saturated process are of particular importance, since the partially saturated process is the most general of the three processes mentioned above. In fact, the envelope statistics in the partially saturated case have been

a) Present address: 153 Alkamenous, Athens 10446, Greece. shown to reduce to the corresponding statistics of the unsaturated and fully saturated cases as they have been derived in Refs. 4-6.

Expressions for the PDFs of the envelope of a partially saturated ocean acoustic process at one ${ }^{1}$ and two ${ }^{2}$ points in time have been recently derived. Both expressions are not in an analytical closed form and their applications require numerical integrations. The evaluation of the upcrossing and downcrossing statistics of a given threshold requires integration of the first- and second-order envelope PDFs. Multiple nested numerical integrations should be performed to evaluate the aforementioned statistics.

The results for the envelope PDFs derived in Refs. 1 and 2 are also of interest in recent research in torsional vibrations and structural reliability. ${ }^{7-9}$ Under certain assumptions, the envelope statistics of the stress in a diesel engine shaft are the same as that of a partially saturated acoustic process. An integration scheme based on Robert's method ${ }^{10}$ has been devised to evaluate the necessary upcrossing statistics in that case.

Since the computational cost of the above evaluation is high, and in the absence of an exact closed-form expression, it is highly desirable to derive approximate, closed-form expressions for the integrals involved in the evaluation of the first- and second-order PDFs of the envelope of a partially saturated process. This will make the detection algorithm more efficient and also provide insight into the limiting behavior of the PDFs.

Finally, it should be mentioned that the importance and usefulness of a closed-form expression for the envelope PDF is not confined to the particular problem in underwater acoustics and structual reliability, but extends into other 
problems in random vibration and statistical communications theory.

The first-order PDF of the envelope of a partially saturated process $\rho$ is ${ }^{1}$

$$
\begin{aligned}
p_{\rho}\left(\rho_{0}\right)= & \frac{\rho_{0}}{2 \pi \sigma_{x} \sigma_{y}\left(1-\rho_{x y}^{2}\right)^{1 / 2}} \\
& \times \exp \left[\frac { - 1 } { 2 ( 1 - \rho _ { x y } ^ { 2 } ) } \left(\frac{\rho_{0}^{2}+2 \mu_{x}^{2}}{2 \sigma_{x}^{2}}\right.\right. \\
& \left.\left.-\frac{2 \rho_{x y} \mu_{x} \mu_{y}}{\sigma_{x} \sigma_{y}}+\frac{\rho_{0}^{2}+2 \mu_{y}^{2}}{2 \sigma_{y}^{2}}\right)\right] I,
\end{aligned}
$$

where

$I=\int_{0}^{2 \pi} \exp (a \cos \phi+b \sin \phi+c \cos 2 \phi+d \sin 2 \phi) d \phi$,

$a=\frac{-\rho_{0}^{2}}{4\left(1-\rho_{x y}^{2}\right)}\left(\frac{1}{\sigma_{x}^{2}}-\frac{1}{\sigma_{y}^{2}}\right), \quad b=\frac{-\rho_{0}^{2}}{2\left(1-\rho_{x y}^{2}\right)} \frac{\rho_{x y}}{\sigma_{x} \sigma_{y}}$,

$c=\frac{-\rho_{0}}{\left(1-\rho_{x y}^{2}\right)}\left(\frac{\rho_{x y} \mu_{y}}{\sigma_{x} \sigma_{y}}-\frac{\mu_{x}}{\sigma_{x}^{2}}\right)$,

and

$d=\frac{-\rho_{0}}{\left(1-\rho_{x y}^{2}\right)}\left(\frac{\rho_{x y} \mu_{x}}{\sigma_{x} \sigma_{y}}-\frac{\mu_{y}}{\sigma_{y}^{2}}\right)$.

In the same equation, $\mu_{x}, \mu_{y}$ are the means and $\sigma_{x}^{2}, \sigma_{y}^{2}$ are the variances of the coefficients of the cosine and sine components of the acoustic signal, respectively. Finally, $\rho_{x y}$ stands for the correlation coefficient of these components.

It is the scope of this article to derive a closed-form, approximate expression for integral I involved in the evaluation of the first-order PDF of the envelope, and, consequently, an expression for that PDF. The exponent of $I$ in (2) is the sum of two sinusoids with a given phase difference. Therefore, it is expected to have four stationary points. The derivation is based on the approximation of the exponent in the neighborhoods of the maxima by second-order polynomials having the same roots and maxima. The derived expression is tested by applying it for different cases and comparing the results with those obtained by direct numerical integration.

\section{DERIVATION OF THE APPROXIMATE CLOSED-FORM EXPRESSION}

Our approach is based on the approximation of the argument of the exponent in (2) in the neighborhoods of the maxima by second-order polynomials having the same roots and maxima. It is then clear that the integral obtained after the approximation can be evaluated in closed form.

We use the following abbreviations:

$$
\begin{aligned}
& F(\phi)=a \cos \phi+b \sin \phi+c \cos 2 \phi+d \sin 2 \phi, \\
& I=\int_{0}^{2 \pi} \exp [F(\phi)] d \phi .
\end{aligned}
$$

The exponent can be written in the following form:

$$
F(\phi)=a^{\prime} \cos \left(\phi+\delta_{1}\right)+b^{\prime} \cos \left(2 \phi+\delta_{2}\right),
$$

where

$$
\begin{aligned}
& a^{\prime}=\left(a^{2}+b^{2}\right)^{1 / 2}, \\
& b^{\prime}=\left(c^{2}+d^{2}\right)^{1 / 2}, \\
& \delta_{1}=\tan ^{-1}(-b / a), \quad \delta_{1} \text { in }[-\pi, \pi], \\
& \delta_{2}=\tan ^{-1}(-d / c), \quad \delta_{2} \text { in }[-\pi, \pi] .
\end{aligned}
$$

First, we find the maxima, minima, and the roots of the exponents $F(\phi)$. For the stationary points, we set $d F /$ $d \phi=0$. That gives

$$
-a \sin \phi+b \cos \phi-2 c \sin 2 \phi+2 d \cos 2 \phi=0 .
$$

Substituting $\cos \phi=u$ and $\sin \phi= \pm \sqrt{1-u^{2}}$, we get

$$
\begin{aligned}
16\left(d^{2}+c^{2}\right) u^{4}+8(b d+a c) u^{3} \\
\quad+\left[a^{2}+b^{2}-16\left(c^{2}+d^{2}\right)\right] u^{2} \\
\quad+(-4 b d-8 a c) u+4 d^{2}-a^{2}=0 .
\end{aligned}
$$

A similar transformation yields the following equation for the roots of the exponent: We set $F=0$. After the substitution $\cos \phi=u$, we obtain

$$
\begin{aligned}
& 4\left(c^{2}+d^{2}\right) u^{4}+4 a c u^{3}+4 b d u^{3} \\
& +\left[a^{2}+b^{2}-4\left(c^{2}+d^{2}\right)\right] u^{2} \\
& \quad-2 a c u-4 b d u+c^{2}-b^{2}=0 .
\end{aligned}
$$

Hence, in both cases, we have an algebraic equation of the fourth order in $u$. The roots and the maxima of the exponent can be found by solving the corresponding algebraic equations with respect to $u$, and, consequently, the equation

$$
\phi_{i}=\cos ^{-1} u_{i}, \quad 0 \leqslant \phi_{i}<2 \pi
$$

for $\phi_{i}$. The last equation has two roots $\phi_{i}$ for each $u_{i}$. Therefore, we have to select those angles that satisfy Eq. (10) for the maxima, and $F(\phi)=0$, where $F(\phi)$ is given by (3), for the roots.

The roots of the fourth-order algebraic equation

$$
u^{4}+\alpha_{1} u^{3}+\alpha_{1} u^{2}+\alpha_{4}=0
$$

are given by the following theorem ${ }^{11}$ :

If $y_{1}$ is a real root of the third-order equation

$y^{3}-\alpha_{2} y^{2}+\left(\alpha_{1} \alpha_{3}-4 \alpha_{4}\right) y+4 \alpha_{2} \alpha_{4}-\alpha_{3}^{2}-\alpha_{1}^{2} \alpha_{4}=0$,

then the four roots of (13) are the roots of the quadratic equation

$$
\begin{gathered}
Z^{2}+\frac{1}{2}\left[\alpha_{1} \pm\left(\alpha_{1}-4 \alpha_{2}+4 y_{1}\right)^{1 / 2}\right] Z \\
+\frac{1}{2}\left[y_{1} \mp\left(y_{1}^{2}-4 \alpha_{4}\right)^{1 / 2}\right]=0 .
\end{gathered}
$$

The roots of the third-order equation $y^{3}+\beta_{1} y^{2}+\beta_{2} y+\beta_{3}=0$ are

$$
\begin{aligned}
& y_{1}=S+T-\frac{1}{3} \beta_{1}, \\
& y_{2}=-\frac{1}{2}(S+T)-\frac{1}{3} \beta_{1}+\frac{1}{2} i \sqrt{3}(S-T), \\
& y_{3}=-\frac{1}{2}(S+T)-\frac{1}{3} \beta_{1}-\frac{1}{2} i \sqrt{3}(S-T),
\end{aligned}
$$

where

$$
\begin{aligned}
& S=\left[R+\left(Q^{3}+R^{2}\right)^{1 / 2}\right]^{1 / 3}, \\
& T=\left[R-\left(Q^{3}+R^{2}\right)^{1 / 2}\right]^{1 / 3}
\end{aligned}
$$


and

$$
Q=\frac{1}{9}\left(3 \beta_{2}-\beta_{1}^{2}\right), \quad R=\frac{1}{34}\left(9 \beta_{1} \beta_{2}-27 \beta_{3}-2 \beta_{1}^{3}\right) .
$$

If $\left[\phi_{1}, F\left(\phi_{1}\right)\right]$ is a positive local maximum of the exponent and $\phi_{1}^{\prime}$ and $\phi_{1}^{\prime \prime}$ are the two roots before and after $\phi_{1}$, respectively, the value of the integral

$$
A_{1}=\int_{\phi_{1}^{\prime}}^{\phi_{1}^{\prime \prime}} e^{F} d \phi
$$

can be approximated by

$$
\int_{-\rho_{1}^{\prime}}^{0} e^{-\lambda^{\prime} x^{2}+\mu} d x+\int_{0}^{\rho_{1}^{\prime \prime}} e^{-\lambda^{\prime \prime} x^{2}+\mu} d x
$$

where

$$
\begin{aligned}
& \rho_{1}^{\prime}=\phi_{1}-\phi_{1}^{\prime}, \quad \mu=F\left(\phi_{1}\right), \quad \lambda^{\prime}=\mu / \rho_{1}{ }^{2}, \\
& \rho_{1}^{\prime \prime}=\phi_{1}^{\prime \prime}-\phi_{1}, \text { and } \lambda^{\prime \prime}=\mu / \rho_{1}^{\prime 2} .
\end{aligned}
$$

Therefore,

$$
A_{1}=e^{\mu}\left(\rho_{1} / 2\right) \sqrt{\pi / \mu} \text { erf }(\sqrt{\mu}) .
$$

In the case of a local minimum, where we do not expect a significant contribution to the value of the total integral, we use a quadratic approximation to the quantity $F$. If $\left[\phi_{2}\right.$, $\left.F\left(\phi_{2}\right)\right]$ is a local minimum and $\mu=F\left(\phi_{2}\right)<0$, the integral

$$
A_{2}=\int_{\phi_{2}^{\prime}}^{\phi_{2}^{\prime \prime}} e^{F} d \phi
$$

where $\phi_{2}^{\prime}$ and $\phi_{2}^{\prime \prime}$ are the roots of the exponent immediately before and after $\phi_{2}$, respectively, can be approximated by

$$
A_{2}=\rho_{2}\left(\frac{1}{3}+e^{\mu}\right) \text {, }
$$

where $\rho_{2}=\phi_{2}^{\prime \prime}-\phi_{2}^{\prime}$. Hence,

$$
I=\sum_{i=1}^{4} A_{i}
$$

where

$A_{i} \cong e^{\mu_{i}}\left(\rho_{i} / 2\right) \sqrt{\pi / \mu_{i}} \operatorname{erf}\left(\sqrt{\mu_{i}}\right), i=1,2,3,4, \quad \mu>0$

and

$$
A_{i} \cong \rho_{i}\left(\frac{1}{3}+e^{\mu_{i}}\right), i=1,2,3,4, \quad \mu \leqslant 0 .
$$

The final result for the first-order PDF then becomes

$$
\begin{aligned}
p_{\rho}\left(\rho_{0}\right) \leqslant & \frac{\rho_{0}}{2 \pi \sigma_{x} \sigma_{y}\left(1-\rho_{x y}^{2}\right)^{1 / 2}} \exp \left[\frac { - 1 } { 2 ( 1 - \rho _ { x y } ^ { 2 } ) } \left(\frac{\rho_{0}^{2}+2 \mu_{x}^{2}}{2 \sigma_{x}^{2}}\right.\right. \\
& \left.\left.-\frac{2 \rho_{x y} \mu_{x} \mu_{y}}{\sigma_{x} \sigma_{y}}+\frac{\rho_{0}^{2}+2 \mu_{y}^{2}}{2 \sigma_{y}^{2}}\right)\right] \sum_{i=1}^{4} A_{i} .
\end{aligned}
$$

Note that it is possible to have only two roots, $\phi_{1}$ and $\phi_{2}$. In this case, we can take $I=A_{1}+A_{2}$. In the case where three stationary points exist between the two roots, we can handle the middle one as if it were another root.

In the frequent case where the maximum value of the exponent is large $(\mu>4)$, Laplace's method may be applied. ${ }^{12}$ According to this method the major contribution to the value of the integral arises from the immediate vicinity of those points of the interval, at which the exponent assumes its largest value. If $F(\phi)$ has more than one maxima, we may break up the integral into a number of integrals, such that in each integral $F(\phi)$ reaches its maximum at one of the end points and at no other point. Accordingly, we shall assume that $F(\phi)$ reaches its maximum at $\phi=\phi_{0}$, and that $F(\phi)<F\left(\phi_{0}\right)$ in the interval discussed. Since our $F$ is twice continuously differentiable, and $F^{\prime}\left(\phi_{0}\right)=0, F^{\prime \prime}\left(\phi_{0}\right)<0$, we may apply the technique introduced by Laplace. Specifically, a new variable $u$ defined by the substitution

$$
F\left(\phi_{0}\right)-F(\phi)=u^{2} F^{\prime}(\phi),
$$

will be negative in $\phi_{0} \leqslant \phi \leqslant \phi_{0}+\eta$, for some sufficiently small $\eta$. As $F(\phi)$ becomes large,

$$
\begin{aligned}
I \cong 2 \int_{\phi_{0}}^{\phi_{0}+\eta} e^{F(\phi)} d \phi \\
=-4 \int_{0}^{U} \frac{u}{F^{\prime}(\phi)}\left\{\exp \left[F\left(\phi_{0}\right)-u^{2}\right]\right\} d u,
\end{aligned}
$$

where

$$
u=\left[F\left(\phi_{0}\right)-F\left(\phi_{0}+\eta\right)\right]^{1 / 2}>0 .
$$

Since only the neighborhood of $u=0$ matters, we may replace $u / F^{\prime}(\phi)$ by $-\left[-2 F^{\prime \prime}\left(\phi_{0}\right)\right]^{-1 / 2}$, which is the limit of $u / F^{\prime}(\phi)$ as $\phi \rightarrow \phi_{0}$, and obtain

$$
I \cong\left(\frac{-2}{F^{\prime \prime}\left(\phi_{0}\right)}\right)^{1 / 2} 2 \int_{0}^{u}\left\{\exp \left[-u^{2}+F\left(\phi_{0}\right)\right]\right\} d u .
$$

By the same argument, we may extend the integration to $u=\infty$ and finally obtain Laplace's result:

$$
I \cong e^{F\left(\phi_{0}\right)}\left[-2 \pi / F^{\prime \prime}\left(\phi_{0}\right)\right]^{1 / 2} \text {. }
$$

In the general case, where we have to deal with two maxima, $\mu_{1}=F\left(\phi_{01}\right)$ and $\mu_{2}=F\left(\phi_{02}\right), \phi \in[0,2 \pi]$, the value of the integral will be

$$
I \cong e^{\mu_{1}}\left[-2 \pi / F^{\prime \prime}\left(\phi_{01}\right)\right]^{1 / 2}+e^{\mu_{2}}\left[-2 \pi / F^{\prime \prime}\left(\phi_{02}\right)\right]^{1 / 2} .
$$

\section{A. Special cases}

Case $A: a^{2}+b^{2} \gg c^{2}+d^{2}$ obtain

Using Eq. (5) and the transformation $\psi=\phi+\delta_{1}$, we

$$
I=\int_{0}^{2 \pi} \exp F_{1}(\psi) d \psi
$$

where $\quad F_{1}(\psi)=a^{\prime} \cos \psi+b^{\prime} \cos (2 \psi+\delta) \quad$ and $\delta=\delta_{2}-2 \delta_{1}$. In this case, we expect the maximum value of $F_{1}(\psi)$ to be near $\psi=0$.

For small values of $\psi$,

$$
a^{\prime} \cos \psi \cong a^{\prime}\left(1-\psi^{2} / 2\right) \text {, }
$$

and

$$
\begin{aligned}
b^{\prime} \cos (2 \psi+\delta) & =b^{\prime}(\cos 2 \psi \cos \delta-\sin 2 \psi \sin \delta) \\
& \cong\left[\left(1-2 \psi^{2}\right) \cos \delta-2 \psi \sin \delta\right] b^{\prime} .
\end{aligned}
$$

Consequently,

$$
\begin{aligned}
F_{1} \cong & a^{\prime}-\left(a^{\prime} / 2\right) \psi^{2}+\left(\cos \delta-2 \psi^{2} \cos \delta-2 \psi \sin \delta\right) b^{\prime} \\
= & a^{\prime}+b^{\prime} \cos \delta-2 \psi b^{\prime} \sin \delta \\
& -\psi^{2}\left(2 b^{\prime} \cos \delta+a^{\prime} / 2\right) .
\end{aligned}
$$


To find the value of $\psi$ that maximizes $F_{1}$, set $d F_{1} / d \psi=0$, or equivalently, $-2 b^{\prime} \sin \delta-4 \psi b^{\prime} \cos \delta+a^{\prime} / 2=0$, which yields

$$
\psi_{0}=-b^{\prime} \sin \delta /\left(2 b^{\prime} \cos \delta+a^{\prime} / 2\right)
$$

( since $a^{\prime} \gg b^{\prime}$ and $\left|\psi_{0}\right| \ll 1$ ).

An approximation to $I$ can now be the quantity $2 \pi I_{0}(\mu)$ for $\mu<4$, where $\mu=\psi\left(\phi_{0}\right), \phi_{0}=\psi_{0}-\delta_{1}$, and $I_{0}(\mu)$ is the modified Bessel function of order 0 . The above approximation is valid for small values of $\mu$. For large values of $\mu$, we can use Eq. (28).

Case B: $a^{2}+b^{2} \ll c^{2}+d^{2}$

After the substitutions $\psi=\phi+\delta_{2} / 2$ and $\delta=\delta_{1}-\delta_{2} /$ 2 , the exponential becomes

$$
F_{2}(\psi)=a^{\prime} \cos (\psi+\delta)+b^{\prime} \cos 2 \psi
$$

The exponent has two maxima located near 0 and $\pi$.

Using a Taylor expansion, we obtain the following approximations for small $\psi$ :

$$
a^{\prime} \cos (\psi+\delta)=a^{\prime}\left[\left(1-\psi^{2} / 2\right) \cos \delta-\psi \sin \delta\right],
$$

and

$$
b^{\prime} \cos 2 \psi \simeq b^{\prime}\left(1-2 \psi^{2}\right) .
$$

Consequently,

$$
F_{2} \simeq a^{\prime} \cos \delta-a^{\prime}\left(\psi^{2} / 2\right) \cos \delta-a^{\prime} \psi \sin \delta+b^{\prime}-2 b^{\prime} \psi^{2} \text {. }
$$

The value of $\psi$ that lies near 0 and maximizes $F_{2}$ is

$$
\begin{aligned}
\psi_{01} & =a^{\prime} \sin \delta /\left(a^{\prime} \cos \delta+4 b^{\prime}\right) \\
& \simeq-a^{\prime} \sin \delta /\left(a^{\prime}+4 b^{\prime}\right) .
\end{aligned}
$$

For the second maximum, we have

$$
\psi_{02}=\pi-\psi_{01} \text {. }
$$

An approximation to $I$ can now be the quantity

$$
2 \pi I_{0}\left(\mu_{1}\right)+2 \pi I_{0}\left(\mu_{2}\right),
$$

where $\mu_{1}$ and $\mu_{2}$ are the maxima of $F$ at $\psi_{01}$ and $\psi_{02}$, respectively. Equation (33) should be used for small values of $\mu_{1}$, $\mu_{2}$. For values of $\mu_{1}, \mu_{2}$ greater than 4, we can use Eq. (29).

Case C:

If parameters $a, b, c, d$ are all small ( $\ll 1$ ), an approximation can be obtained by expanding the exponent, in power series about zero, integrating term by term and dropping the higher than second-order terms:

$$
e^{F}=1+(F / 1 !)+\left(F^{2} / 2 !\right)+\cdots,
$$

hence,

$$
\begin{aligned}
I & =\int_{0}^{2 \pi} e^{F} d \phi \cong \int_{0}^{2 \pi}\left(1+F+\frac{F^{2}}{2}\right) d \phi \\
& =\left[4+\left(a^{2}+b^{2}+c^{2}+d^{2}\right)\right] \pi / 2
\end{aligned}
$$

\section{DERIVATION OF A BESSEL SERIES APPROXIMATION}

The first-order PDF can be evaluated in terms of an infinite power series of modified Bessel functions. The evaluation is based on the expansion of the cosine terms of the exponential into power series as follows: $a^{\prime} \cos \left(\phi+\delta_{1}\right)=\sum_{n=0}^{\infty} \epsilon_{n}(-1)^{n} I_{n}\left(a^{\prime}\right) \cos \left(n \phi+n \delta_{1}\right)$

and

$$
\begin{aligned}
b^{\prime} \cos \left(2 \phi+\delta_{2}\right) \\
=\sum_{m=0}^{\infty} \epsilon_{m}(-1)^{m} I_{m}\left(b^{\prime}\right) \cos \left(2 m \phi+m \delta_{2}\right),
\end{aligned}
$$

where

$\epsilon_{n}(m)=1$ for $n(m)=0$ and $\epsilon_{n}(m)=2$ for $n(m) \geqslant 1$.

Substituting (35) and (36) in (4), we obtain

$$
\begin{aligned}
I= & \int_{0}^{2 \pi} \sum_{n=0}^{\infty} \sum_{m=0}^{\infty} \epsilon_{n} \epsilon_{m}(-1)^{m+n} I_{n}\left(a^{\prime}\right) I_{m}\left(b^{\prime}\right) \\
& \times \cos \left(n \phi+n \delta_{1}\right) \cos \left(2 m \phi+m \delta_{2}\right) d \phi,
\end{aligned}
$$

or

$$
\begin{aligned}
I= & \frac{1}{2} \sum_{n_{1}=1}^{2} \sum_{n=0}^{\infty} \sum_{m=0}^{\infty} \int_{0}^{2 \pi} \cdot \epsilon_{m} \epsilon_{n}(-1)^{m+n} I_{n}\left(a^{\prime}\right) \\
& \times I_{m}\left(b^{\prime}\right) \cos \left\{\left[2 m+(-1)^{\left.n_{1} n\right] \phi}\right.\right. \\
& \left.+\left[m \delta_{2}+(-1)^{n_{1}} n \delta_{1}\right]\right\} d \phi .
\end{aligned}
$$

The only terms of the sum in (37) that are not zero are for $n=2 m$ and $n_{1}=1$. Dropping all the terms that are zero, Eq. (37) yields

$$
\begin{aligned}
I= & \pi I_{0}\left(a^{\prime}\right) I_{0}\left(b^{\prime}\right)+\pi \sum_{m=1}^{\infty} \epsilon_{m} \epsilon_{2 m}(-1) I_{m}\left(a^{\prime}\right) I_{2 m}\left(b^{\prime}\right) \\
& \times \cos m\left(\delta_{2}-2 \delta_{1}\right) .
\end{aligned}
$$

In most cases, three or four terms in the sum of Eq. (38) are enough to obtain a reasonable estimate of $I$. However, the evaluation of the modified Bessel functions requires a considerable amount of computational effort. To obtain the envelope PDF, we substitute the expression for $I$ as obtained from (38) in (1).

\section{EXAMPLES}

\section{A. General case}

Quantities $a^{2}+b^{2}$ and $c^{2}+d^{2}$ are of the same order of magnitude. Two numerical examples will be considered

\section{1. $a=b=c=d=1$}

Equation (12) becomes

$$
8 u^{4}+8 u^{3}-6 u^{2}-6 u=0,
$$

or

$$
u\left(4 u^{2}-3\right)(u+1)=0,
$$

whose roots are

$$
u_{1}=0, \quad u_{2}=-1, \quad u_{3}=\sqrt{3 / 4}, \quad u_{4}=-\sqrt{3 / 4} .
$$

Since $u=\cos \phi$, the corresponding values of $\phi$ are

$$
\begin{aligned}
& \phi_{1}=\pi / 2=1.5708, \quad \phi_{2}=\pi=3.1416, \\
& \phi_{3}=3.6652, \quad \phi_{4}=5.7596 .
\end{aligned}
$$

Equation (11) becomes

$$
u^{4}+0.5 u^{3}-0.9375 u^{2}-0.3750 u+0.0938=0 .
$$


The third-order equation,

$y^{3}-\alpha_{2} y^{2}+\left(\alpha_{1} \alpha_{3}-4 \alpha_{4}\right) y+\left(4 \alpha_{2} \alpha_{4}-\alpha_{3}^{2}-\alpha_{1}^{2} \alpha_{4}\right)=0$,

becomes

$$
y^{3}+0.9375 y^{2}-0.5625 y-0.5156=0 .
$$

The real root of this equation is $y_{1}=\mathbf{0 . 7 4 5 4}$.

The quadratic equation,

$$
\begin{gathered}
Z^{2}+\frac{1}{2}\left(\alpha_{1} \pm \sqrt{\alpha_{1}^{2}-4 \alpha_{2}+4 y_{1}}\right) Z \\
+\frac{1}{2}\left(y_{1} \pm \sqrt{y_{1}^{2}-4 \alpha_{4}}\right)=0,
\end{gathered}
$$

becomes

$$
\text { (1) } Z^{2}+1.5711 Z+0.5851=0
$$

with roots $Z_{1}=-0.6008$ and $Z_{2}=-0.9644$, and

(2) $Z^{2}-1.0711 Z+0.1602=0$,

with roots $Z_{3}=0.1798$ and $Z_{4}=0.8914$. Since $\phi_{i}=\cos ^{-1} u_{i}, \phi_{i} \in(0,2 \pi)$, we have

$$
\begin{array}{ll}
\phi_{1}=0.4704, & \phi_{2}=2.2228, \\
\phi_{3}=3.4092, & \phi_{4}=4.8932,
\end{array}
$$

and

$$
\begin{array}{ll}
\mu_{1}=F\left(\phi_{1}\right)=2.7418, & \mu_{2}=F\left(\phi_{2}\right)=-1.0402, \\
\mu_{3}=F\left(\phi_{3}\right)=0.1414, & \mu_{4}=F\left(\phi_{4}\right)=-2.0930 .
\end{array}
$$

Since the values of $\mu_{i}$ are small ( $<4$ ), we use (19) and (20):

$$
\begin{aligned}
& A_{1}=e^{\mu_{1}}\left(\rho_{1} / 2\right) \sqrt{\pi / \mu_{1}} \operatorname{erf}\left(\sqrt{\mu_{1}}\right)=15.6523, \\
& A_{2}=\rho_{2}\left(\frac{1}{3}+e^{\mu_{2}}\right)=1.0850, \\
& A_{3}=0.3979, \quad A_{4}=0.9563,
\end{aligned}
$$

and

$$
I=\sum A_{i}=18.0915 .
$$

The exact value of $I$, calculated numerically, is 17.7622 , and, hence, the difference is only $-1.854 \%$.

\section{2. $a=b=c=d$}

Equation (11) is the same as in Sec. III A 1. Hence, $\phi_{01}=0.4704, \mu_{1}=13.7090$, and $F^{\prime \prime}\left(\phi_{01}\right)=-34.6665$. Also,

$$
\phi_{02}=3.4092, \quad \mu_{2}=0.7070,
$$

and

$$
F^{\prime \prime}\left(\phi_{02}\right)=-21.2595 \text {. }
$$

Using (29), we obtain $I \cong 382719$. The exact value of $I$ is 387820 , and, hence, the error is $+1.315 \%$.

\section{B. Special case A: $a^{2}+b^{2} \gg c^{2}+d^{2}$}

Assuming $a=10, b=1, c=1, d=1$, we obtain

$a^{\prime}=\sqrt{a^{2}+b^{2}}=10.0499$,

$\delta_{1}=\tan ^{-1}(-b / d)=-0.0997$.

$b^{\prime}=\sqrt{c^{2}+d^{2}}=1.4142$,

$$
\begin{aligned}
& \delta_{2}=\tan ^{-1}(-d / c)=-0.7854 \\
& \delta=\delta_{2}-2 \delta_{1}=-0.5860 \\
& \psi_{0}=-b^{\prime} \sin \delta /\left(2 b^{\prime} \cos \delta+a^{\prime} / 2\right)=0.1060 \\
& \phi_{0}=\psi_{0}-\delta_{1}=0.2057 \\
& \mu=F\left(\phi_{0}\right)=11.3099 \\
& e^{\mu}=81,685, \text { and } F^{\prime \prime}\left(\phi_{0}\right)=-15.2591
\end{aligned}
$$

Using (28), we obtain $I \cong 52416$. The exact value is $I=53361$, and the error is $+1.771 \%$.

\section{Special case B: $a^{2}+b^{2} \ll c^{2}+d^{2}$}

For $a=1, b=1, c=1$, and $d=10$,

$$
\begin{aligned}
& a^{\prime}=\sqrt{a^{2}+b^{2}}=1.4142, \\
& \delta_{1}=\tan ^{-1}(-b / a)=-0.7854, \\
& b^{\prime}=\sqrt{c^{2}+d^{2}}=10.0499, \\
& \delta_{2}=\tan ^{-1}(-d / c)=-1.4711, \\
& \delta=\delta_{1}-\delta_{2} / 2=-0.0498 .
\end{aligned}
$$

For the first maximum we use Eq. (28):

$$
\psi_{01}=-a^{\prime} \sin \delta /\left(a^{\prime}+4 b^{\prime}\right)=0.0017,
$$

or

$$
\begin{aligned}
& \phi_{01}=\psi_{01}-\delta_{2} / 2=0.7373, \\
& \mu_{1}=11.4624, \quad F^{\prime \prime}\left(\phi_{01}\right)=-41.6118 .
\end{aligned}
$$

For the second maximum,

$$
\psi_{02}=-\psi_{01}=-0.0017
$$

or

$$
\begin{aligned}
& \phi_{02}=\psi_{02}-\delta_{2} / 2+\pi=3.8755, \\
& \mu_{2}=8.6371, \quad F^{\prime \prime}\left(\phi_{02}\right)=-38.7869 .
\end{aligned}
$$

Using formula (29) we get $I \sim 39212$. The exact value of $I$ is 39702 and, hence, the error is $1.233 \%$.

\section{FURTHER RESEARCH}

An approximate closed-form expression for the joint PDF of the envelope at two distinct points in time is a desirable future research direction. The evaluation of this PDF involves two nested integrations. The quantity to be integrated is an exponential function and the exponent $F\left(\phi_{1}, \phi_{2}\right)$ is a generalized form of $F(\phi)$ as defined in Eq. (3). Laplace's method has been applied to integrals depending on two large variables by Fulks ${ }^{13}$ and Thomsen ${ }^{14}$ and to double and multiple integrals by $\mathrm{Hsu}^{15-17}$ and Rooney. ${ }^{18}$ If the stationary points of the exponent $F\left(\phi_{1}, \phi_{2}\right)$ can be analytically located, an approximate closed form expression may be derived.

'P. N. Mikhalevsky, "Envelope Statistics of Partially Saturated Processes," J. Acoust. Soc. Am. 72, 151-158 (1982).

${ }^{2}$ E. Nikolaidis and A. N. Perakis, "Second-Order Statistics and Detection Modeling for Partially Saturated Processes," J. Acoust. Soc. Am. 77, 1078-1085 (1985).

${ }^{3}$ H. N. Psaraftis and A. N. Perakis, "A Sequential Hypothesis Testing, Optimal Stopping Problem in Underwater Acoustic Detection," J. Acoust. Soc. Am. 75, 859-865 (1984). 
${ }^{4}$ P. N. Mikhalevsky, "First-Order Statistics for Finite Bandwidth Multipath Signals with and without Frequency or Phase Modulation," J. Acoust. Soc. Am. 66, 751-762 (1979).

${ }^{5}$ H. N. Psaraftis, A. N. Perakis, and P. N. Mikhalevsky, "New Models for the Ocean Acoustic Detection Process," J. Acoust. Soc. Am. 69, 1724 1734 (1981).

${ }^{6}$ A. N. Perakis and H. N. Psaraftis, "Discrete-Time Detection Modeling for Unsaturated Ocean Acoustic Propagation," J. Acoust. Soc. Am. 74, 1630-1633 (1983).

${ }^{7}$ E. Nikolaidis, A. N. Perakis, and M. G. Parsons, "Structural Reliability of Marine Diesel Engine Propulsion Shafting Systems," presented at the 93rd SNAME Annual Meeting, November 1985, and included in the Transactions, SNAME 93, 189-223 (1985).

${ }^{8}$ N. Nikolaidis, A. N. Perakis, and M. G. Parsons, "Probabilistic Torsional Vibration Analysis of a Motor Ship Propulation Shafting System: The In-
put-Output Problem," J. Ship. Res. 31 (1), 41-52 (1987).

${ }^{9}$ E. Nikolaidis, A. N. Perakis, and M. G. Parsons, "Probabilistic Torsional Vibration Analysis of a Marine Diesel Engine Shafting System: The Level Crossing Problem," J. Appl. Mech. (submitted for publication).

${ }^{10} \mathrm{G}$. Dahlquist and A. Bjorck, Numerical Methods, translated by N. Anderson (Prentice-Hall, Englewood Cliffs, NJ, 1974).

"'R. Spiegel, Mathematical Handbook of Formulas and Tables (McGrawHill, New York, 1967).

${ }^{12}$ A. Erdelyi, Asymptotic Expansions, (Dover, New York, 1955).

${ }^{13}$ W. B. Fulks Proc. Am. Math. Soc. 2, 613-622 (1951).

${ }^{14}$ D. L. Thomsen, Jr., Proc. Am. Math. Soc. 5, 526-532 (1954).

${ }^{15}$ L. C. Hsu, Am. J. Math. 70, 698-708 (1948).

${ }^{16}$ L. C. Hsu, Duke Math. J. 15, 623-632 (1948).

${ }^{17}$ L. C. Hsu, Am. J. Math. 73, 625-634 (1951).

${ }^{18}$ P. G. Rooney, Trans. R. Soc. Can. Sec. 3 47, 29-34 (1953). 